\title{
Pulse height-length analysis of data from neutron monitors DOMC/DOMB with a new data acquisition system
}

\author{
Markus Similä, ${ }^{a}$ Stepan Poluianov, ${ }^{a, *}$ Ilya Usoskin, ${ }^{a}$ Alexander Mishev, ${ }^{a}$ Gennady \\ Kovaltsov $^{b}$ and Du Toit Strauss ${ }^{c}$ \\ a University of Oulu, \\ 90014 Oulu, Finland \\ ${ }^{b}$ Ioffe Physical-Technical Institute, \\ St. Petersburg, Russia \\ ${ }^{c}$ North-West University, \\ Potchefstroom, South Africa \\ E-mail: stepan.poluianov@oulu.fi
}

\begin{abstract}
Two high-altitude polar neutron monitors DOMC and DOMB (Dome C, Concordia station, Antarctic plateau, $3233 \mathrm{~m}$ a.s.1.) received a major electronics upgrade in 2019. While a typical standard neutron monitor data acquisition (DAQ) system only registers the number of pulses from a cosmicray particle detector, the new system digitizes all pulses with $2 \mathrm{MHz}$ sampling rate and stores this information in raw data files. This feature makes it possible to conduct a pulse height-length analysis of the neutron monitor data on a routine basis. In this study, we have analysed several months of the cosmic-ray data recorded with the new DAQ system during 2019-2020 (more than 10 million pulses). We identified several pulse branches corresponding to different processes: (a) secondary particles from individual cosmic-ray cascades, (b) noise, (c) double pulses originated from particles of the same local cascade, (d) high multiple pulses likely related to atmospheric muons, (e) double pulses potentially caused by contamination by neutrons scattered in the neighbouring instrument. We also studied the waiting time distributions of pulses and have shown that two peaks can be clearly distinguished: (1) at about 1 millisecond, which is related to the intracascade particles, and (2) at 30-1000 milliseconds related to different uncorrelated cosmic-ray cascades. Our conclusions are supported by theoretical estimates of the waiting times in different scenarios.
\end{abstract}

$37^{\text {th }}$ International Cosmic Ray Conference (ICRC 2021)

July 12 th - 23rd, 2021

Online - Berlin, Germany

\footnotetext{
${ }^{*}$ Presenter
} 


\section{Introduction}

Neutron monitors (NMs) are standard instruments to measure variability of cosmic rays near the Earth [1]. They are designed to register (count) the hadronic component of the atmospheric cosmicray cascade, mainly secondary neutrons, but also other particles. NMs are energy-integrating detectors, but the worldwide network built of them is used as a global spectrometer, thanks to different geomagnetic cut-off properties of NM locations [2]. Along with the count rate, the multiplicity of NM counts, which can be a rough index of spectral hardness of cosmic rays, is studied [e.g., 3, 4].

This work is based on data from two NMs DOMC and DOMB [5] located at Dome C (75 06' $\mathrm{S}$, $123^{\circ} 23^{\prime}$ E, $3233 \mathrm{~m}$ a.s.l., Antarctic Plateau). DOMC is a mini-NM with a standard design including a lead layer ("producer") that increases probability of registration of high-energy neutrons. DOMB is very similar to DOMC, but "bare", i.e., without lead. This makes it more sensitive to lower-energy neutrons than DOMC. The instruments are in operation since 2015, and in 2019-2020 they got a major upgrade with new data acquisition systems. The new system is based on a single-board computer Raspberry Pi 3 with GNU/Linux. Many new advantages of the new design are not discussed here, see [6] for full detail. We focus at the advanced registration of signals from the particle detector. The system is equipped with analog-to-digital converter (ADC), which digitizes the profile of each individual pulse (corresponding to a particle passing the detector) at $2 \mathrm{MHz}$ sampling rate, and therefore measures its height and length on routine basis. DOMC and DOMB have built-in pulse height and length thresholds, which are set at $0.2 \mathrm{~V}$ and $2.5 \mu$ s as default. NMs ignore pulses that do not exceed these values.

Here, we present statistics of pulses acquired with new electronics of DOMC and DOMB. We analysed the pulse shape, duration and height, and showed that it is possible to study details of the cosmic-ray cascades and their variability with NMs at a new level.

\section{Pulse height-length analysis}

In this work, we analysed data of DOMC and DOMB from 01 January 2020 to 31 May 2020 (about $3 \cdot 10^{8}$ pulses) and from 13 August 2019 to 31 October 2019 (about $4 \cdot 10^{7}$ pulses), respectively. These periods are characterised by quiet solar conditions. The variability of total count rates (corrected to the atmospheric pressure) due to heliospheric modulation of galactic cosmic rays was within $\pm 1 \%$ for both NMs. No solar energetic particle events happened during the studied period. We used the following parameters of pulses: height $A$ (in $\mathrm{V}$ ), length $t$ (duration of a pulse with voltage over the height threshold, in $\mu \mathrm{s}$ ), the $e$-folding decay time $\tau$ of the exponential decline after maximum (in $\mu \mathrm{s}$ ), the waiting time $\Delta T$ between fronts of two neighbouring pulses (in $\mu \mathrm{s}$ ).

Figure 1 shows the pulse height-length histograms for DOMC and DOMB. We identified several clusters of pulses with similar heigh-length properties in both data sets. They are marked with letters A-E in Figure 1 and called "branches" henceforth. The statistics of branches is summarized in Table 1. 
Table 1: Percentage (\%) of pulses in the identified branches.

\begin{tabular}{lcc}
\hline Branch & DOMC & DOMB \\
\hline A (main): normal single pulses & 90.98 & 91.49 \\
B: noise & $5.20^{\mathrm{a}}$ & $7.84^{\mathrm{a}}$ \\
C: multiple intra-cascade pulses & 3.15 & 0.23 \\
D: (possibly) muons & 0.68 & 0.16 \\
E: (possibly) contamination from neighbouring instrument & - & 0.28 \\
\hline
\end{tabular}

${ }^{\mathrm{a} C}$ Conservative upper bound including also pulses from branch A.

\subsection{Branch A: normal pulses}

Pulses of this branch form the dominant fraction $(\sim 91 \%)$ of all pulses registered by DOMC and DOMB. They last 3-22 $\mu$ s and have steep front (a few $\mu \mathrm{s}$ ) and exponential decrease with the decay time $\tau \approx 8 \mu \mathrm{s}$. The typical shapes are shown in Figure 1A. Height $A$ is in a wide range from 0.4 to $3 \mathrm{~V}$, though pulses with $A<0.5 \mathrm{~V}$ are mixed with noise (branch B). Therefore, we limit the normal pulses of branch $\mathrm{A}$ at $\geq 0.5 \mathrm{~V}$. The count rate of this branch is perfectly corrected with the atmospheric pressure. The corrected count rate has low variability ( $\pm 1 \%$ for DOMC and DOMB) in conditions of low solar activity during the studied period. This provided a sufficient proof to identify pulses of branch A as caused by cascade nucleons and reflecting the general cosmic ray variability as intended by design of the instruments.

\subsection{Branch B: noise}

Pulses in this branch have low height $(A<0.5 \mathrm{~V})$ and no definite shape (Figures 1B). The pulses form about $5 \%$ and $7 \%$ of total counts of DOMC and DOMB, respectively. They can be easily rejected by a height threshold set at $0.5 \mathrm{~V}$, which reduces their fraction down to less than $1 \%$ in the total count rate. The count rate of this branch does not show any pronounced pattern in time variability and is clearly not correlated to the atmospheric pressure nor other branches. We conclude that the pulses in branch B are electronic noise.

\subsection{Branch C: intra-cascade pulses}

This branch contains moderately high pulses (higher than 1.0 and 1.5 V for DOMC and DOMB, respectively) with long duration ( $>22 \mu \mathrm{s}$ and $22-32 \mu \mathrm{s}$ for DOMC and DOMB, respectively). The pulses consist mostly of two sub-peaks (Figure 1C). The shape, height, duration and decay time of sub-pulses are very similar to pulses of branch A. The sub-pulses are separated by 3-20 $\mu$ s, which is much longer than the time expected for particles from a local cascade inside the neutron monitor (estimated as an order of a few nanoseconds). However, this time is consistent with the lower part of the waiting time distribution (see detail below) relevant to the atmospheric cascade. When the waiting time is too short for the first pulse to get dropped under the height threshold, the system registers the second (or even third) subsequent pulse as a part of the first one. This is possible for pulses of closely following particles originating from the same atmospheric cascade. The branch is clearly seen in DOMC data $(\sim 3 \%)$, but weak in DOMB $(0.23 \%)$. The count rate of branch C 
shows even stronger atmospheric pressure dependence than branch $\mathrm{A}$. We note that the standard electronics of of neutron monitors type NM64 with the dead time of about $20 \mu$ s makes this kind of pulses indistinguishable from regular ones of banch A.

\subsection{Branch D: (possibly) muons}

Very high long-lasting pulses ( $A=3.0-3.3 \mathrm{~V}$, near the upper limit of ADC, $t=10-60 \mu \mathrm{s}$ ), form this branch. The shape often has a saturation plateau (Figure 1D). There are hints that the pulses are formed by a process different from branches $\mathrm{A}$ and $\mathrm{C}$. We suggest direct generation of ions by cascade muons in the gas $\left(\mathrm{BF}_{3}\right)$ of detectors as a possible explanation of the branch. Propagation of a charged muon can cause several electromagnetic avalanches inside the detector tube, which can last longer and be recorded as a single high-magnitude long-duration pulse. This process is different from the well-studied muon contribution via local production of neutrons in the lead layer and their consequent standard registration by the detector tube [7]. In this case, an incident muon causes a regular pulse in the NM data and therefore cannot be distinguished from incident nucleons (branches A and C). Thus, we can suggest that branch D is related to non-hadronic particles. However, this needs a more detailed study out of the scope of this work.

The branch shows strong variability of its count rate in time $( \pm 25 \%$ and $\pm 5 \%$ in DOMC and DOMB, respectively, see Figure 2). It can be explained by change of the atmospheric height. Figure 2 confirms this suggestion with the plotted count rate of branch D and geopotential height $\Delta_{100}$ of the atmospheric level $100 \mathrm{hPa}$ (roughly the height of muon production). These two curves have a good agreement $\left(\mathrm{r}=-0.84, p\right.$-value $\left.<10^{-6}\right)$. It confirms that cascade muons are likely responsible for the pulses in branch D. Thus, there is an opportunity to use DOMC/DOMB as muon detectors (though with low efficiency) with a high lower threshold $V_{0}>3.0 \mathrm{~V}$.

\subsection{Branch E: (possible) contamination from DOMC}

This branch exists only in DOMB data (Figure 1E) making just $0.28 \%$ of all counts. The pulses have normal height $(A=1.0-2.5 \mathrm{~V})$ and double length $(t=30-60 \mu \mathrm{s})$. Their shape typically looks like two sub-pulses, though there are three-pulse cases for duration $>50 \mu \mathrm{s}$. We do not have clear explanation of this branch, but we can speculate that it is contamination from DOMC (and its lead part) standing about 1 meter away from DOMB. Tertiary neutrons produced in lead can reach DOMB and be registered there. Since their propagation is diffusive (takes about $20 \mu \mathrm{s}$ to cross $1 \mathrm{~m}$ of air and moderator/reflector of DOMB), several neutrons can arrive to DOMB at slightly different times, though still close enough to form a double-peak long pulse.

\section{Waiting time analysis}

With new precise timing, we analysed the waiting time distributions of pulses recorded by DOMC and DOMB. The waiting time $\Delta T$ is the time interval (in $\mu \mathrm{s}$ ) between fronts of two consecutive pulses. Here we analysed only waiting times $\Delta T>100 \mu$ s. The results are shown as $\log$-binned histograms for different pulse height thresholds $V_{0}$ (i.e., $A \geq V_{0}$ ) in Figure 3. In general, the waiting time distributions keep similar shape for different $V_{0}$. There are two separated clusters of pulses denoted as peak I and peak II. Peak I is located at $\Delta T$ of $0.5-5 \mathrm{~ms}$, while peak II is between $10 \mathrm{~ms}$ and $10 \mathrm{~s}$. 

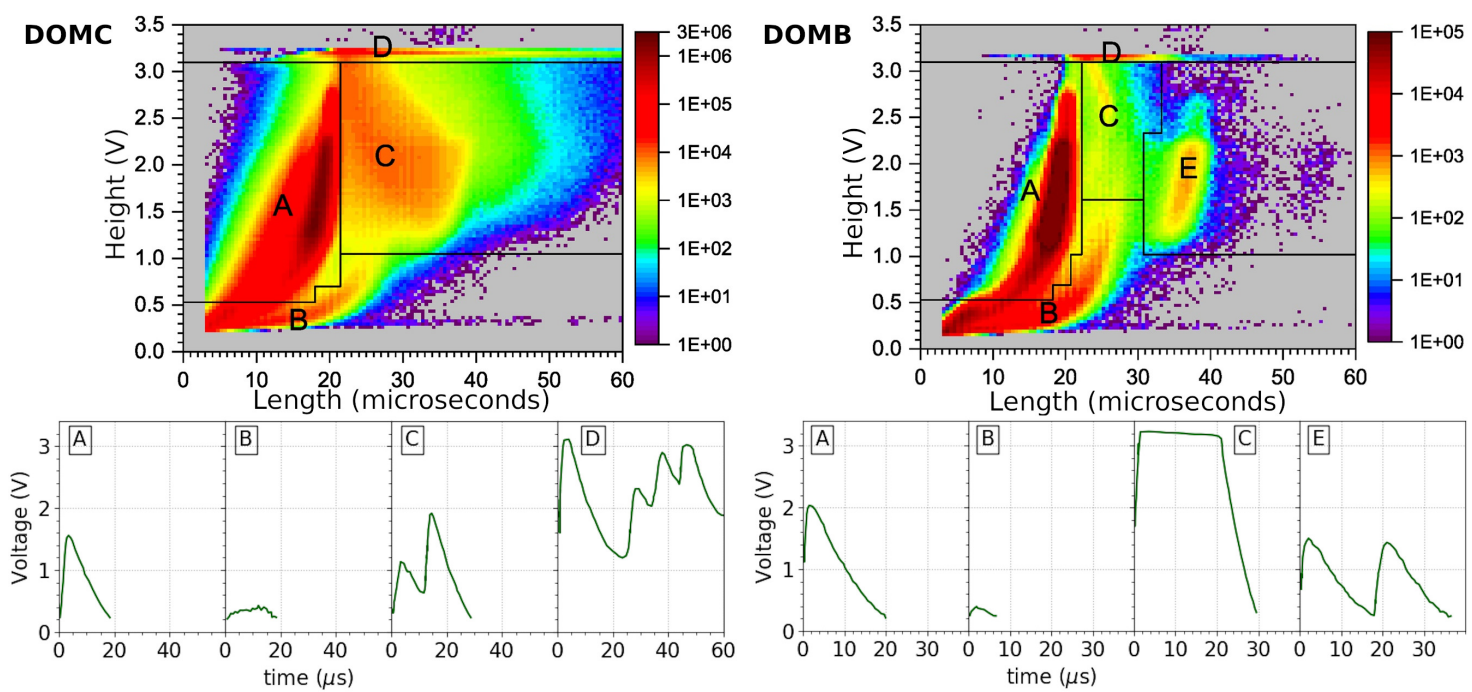

Figure 1: NM pulses: the length-height distributions (upper panels) and typical time profiles (lower panels). Left and right sides correspond to DOMC and DOMB, respectively. Letters A-E indicate branches discussed in the text.

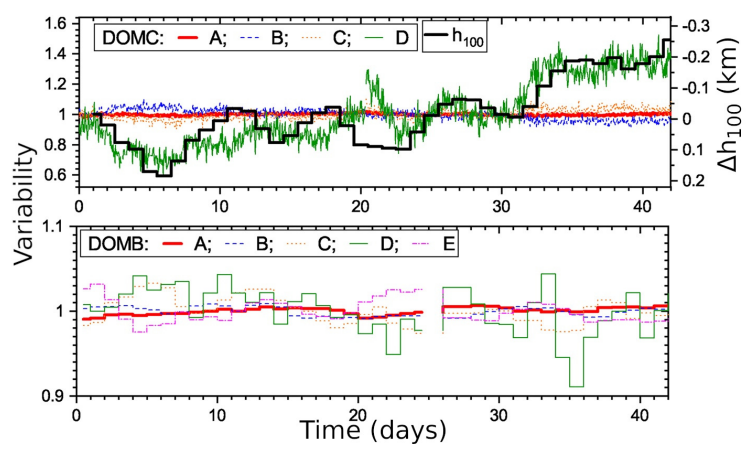

Figure 2: Time variability of count rates of different branches in DOMC and DOMB data (top and bottom panels, respectively). The thick black line indicates variability of the geopotential height $\Delta_{100}$ of the atmospheric level $100 \mathrm{hPa}$, it axis is shown on the right.

\subsection{Peak I: Intra-cascade pulses}

Peak I keeps its stable shape and location at $0.5-5 \mathrm{~ms}$ over $V_{0}$ from 0.2 to $2.5 \mathrm{~V}$ for both DOMC and DOMB. It makes from $35 \%$ (default $V_{0}=0.2 \mathrm{~V}$ ) to $2.5 \%\left(V_{0}=2.5 \mathrm{~V}\right.$ ) for DOMC and from $7 \%$ to $0.1 \%$ for DOMB (with similar $V_{0}$, see Figure 3). We interpret the peak as a contribution from nucleons, mainly neutrons, originating from the same atmospheric cascade. Although secondaries generally form a relatively thin front of the cascade and propagate within a narrow time window, secondary neutrons diffuse. Thus, neutrons have relatively broader distribution of their arrival times.

We did a Monte Carlo simulation of the cascade propagation with Geant4 v.10.6.0 [8] to verify our interpretation of peak I. We modelled 1 million protons, which impinged the realisticallysized atmosphere (NRLMSISE-00 [9]) vertically. The protons had $10 \mathrm{GeV}$ of energy, which approximately equals the effective energy of galactic cosmic rays for a polar NM [10]. The model 

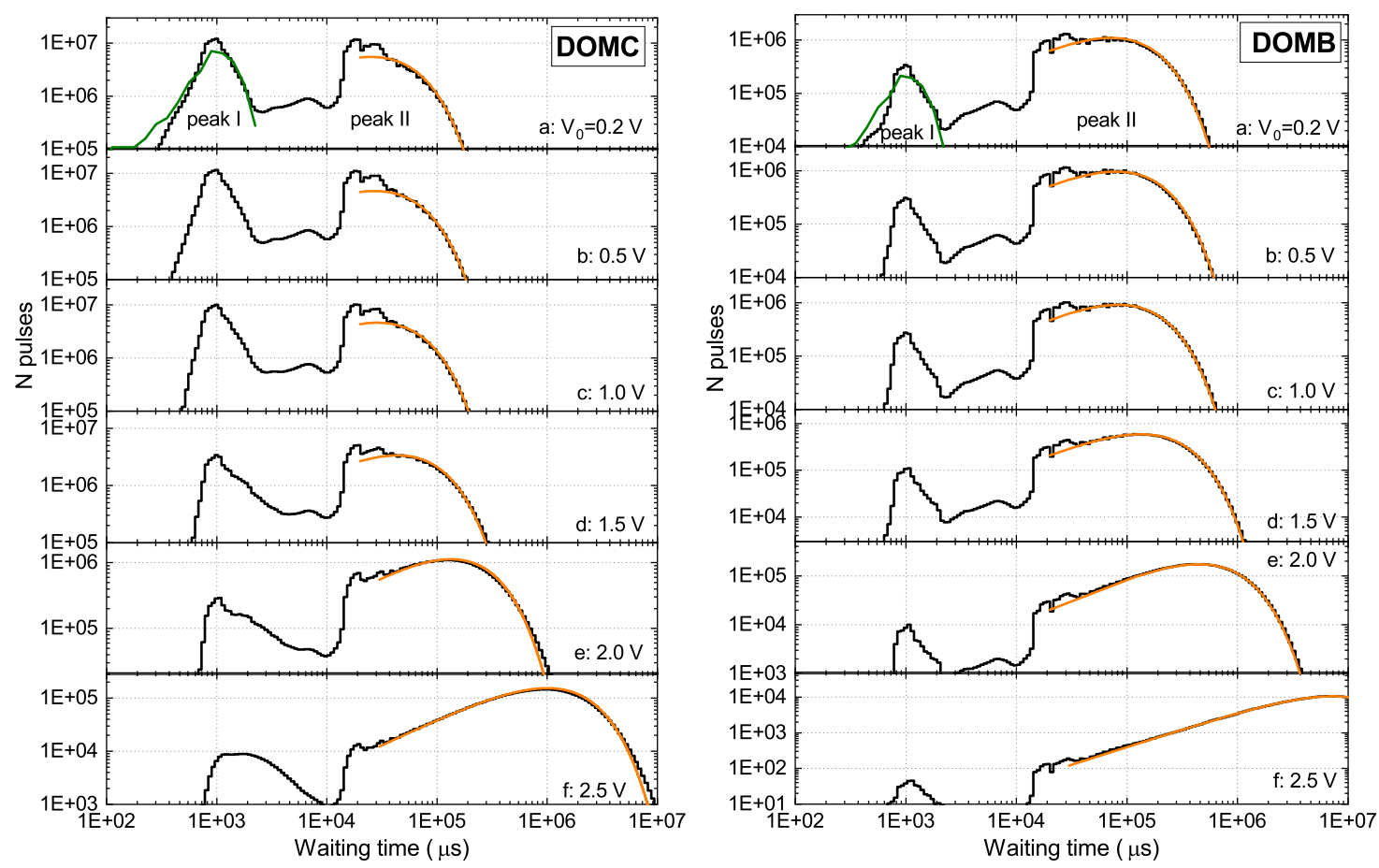

Figure 3: Waiting time $\Delta T$ distribution of pulses registered by DOMC (left) and DOMB (right). The NM data are shown as grey log-binned histograms. Panels from a to $\mathrm{f}$ correspond to different height thresholds $V_{0}$ from default $0.2 \mathrm{~V}$ to $2.5 \mathrm{~V}$ (see the legends). The green lines represent the results of the Monte Carlo simulation of the waiting time between particles originating from the same cascade. The orange lines are theoretical waiting times calculated from randomly occurring counts representing independent cosmic-ray cascades, see details in the text. Marks I and II indicate peaks I and II caused by intra-cascade and inter-cascade particles registered by NMs, respectively.

tracked secondary neutrons crossing the level corresponding to elevation of DOMC and DOMB (650 $\mathrm{g} / \mathrm{cm}^{2}$ of depth), The energy, coordinates and timestamp of each crossing were stored and analysed. This allowed us to estimate the waiting time $\Delta T$ of epithermal $(\leq 10 \mathrm{keV})$ neutrons crossing the level within $1 \mathrm{~m}$ of distance from each other and originating from the same cascade. The log-binned waiting time data obtained from simulations is plotted in Figure 3 as green lines. Note, the lines were scaled in Y-axis to match the experimental waiting time distribution. One can see that the waiting times of peak I based on the NM data agrees with the waiting times of the modelled curve very well for both DOMC and DOMB. Therefore, we concluded that peak I is caused neutrons originating from the same cosmic-ray cascade in the atmosphere. The multiplicity (number of registered particles coming from the same cascade [e.g., 3, 4]) is related to pulses belonging to peak I. DOMC and DOMB show the multiplicity values of 1.5 and 1.06 in the discussed data sets, respectively (with default $V_{0}=0.2 \mathrm{~V}$ ).

\subsection{Peak II: Inter-cascade pulses}

Peak II is formed by pulses with significantly longer waiting times (10 ms $-10 \mathrm{~s})$. In contrast to peak I, it is formed by particles from different cosmic-ray cascades. We tested this with a theoretical estimate of the waiting time $\Delta T$ distribution of independent cascades (orange lines in Figure 3). 
The modelled $\Delta T$ were calculated in assumption of independent randomly occurring pulses with the mean rate equal to the count rates of DOMC and DOMB with a given height threshold $V_{0}$. The result calculated for a log-binned histogram perfectly describes peak II, as shown in Figure 3, and proves our interpretation of the peak as related to pulses from different individual cascades.

\section{Discussion and summary}

We have shown a pulse height-length analysis of data collected with DOMC and DOMB neutron monitors in the sections above. The standard neutron monitor is an energy-integrating detector and, therefore, cannot measure the cosmic-ray spectrum being along. Because of that, NMs are placed in locations with different geomagnetic cut-off properties and form a worldwide network, which, in turn, can be used as a global spectrometer [2]. During solar energetic particle events, especially causing weak GLEs (ground-level enhancements) and sub-GLEs [11], only a few NMs show non-zero responses. Analyses of such events are very limited in acquired data, and any additional information on spectral properties of registered particles is extremely valuable. The finding of this work that muon-induced counts can be separated from ones caused by nucleons, and the detailed pulse analysis overall make it potentially possible to use DOMC, DOMB and other NMs with similar data acquisition systems as particle spectrometers, though with limited quality of estimates. This would be a significant contribution to the existing capabilities of the global NM network and would notably improve quality of reconstructions of weak GLE and sub-GLE events.

In conclusion, we summarized this work in the following statements:

- DOMC and DOMB got a new data acquisition system, and a large number of individual pulses from cosmic-ray detectors were routinely recorded: their profiles sampled at 2-MHz rate, heights, lengths and precise time stamps.

- The height-length analysis revealed several branches of pulses in DOMC and DOMB data: (A) normal pulses associated with secondary nucleons from individual cascades; (B) noise signals; (C) double pulses from closely coming (in time) particles of the same cascade; (D) pulses from (likely) cascade muons; (E, DOMB only) pulses probably caused by contamination of the neighbouring instrument.

- The waiting time analysis showed two clearly separate peaks at about 1 and $50 \mathrm{~ms}$. They correspond to particles originating from the same cosmic-ray cascade and defining the multiplicity (peak I) and to particles coming from different independent cascades (peak II).

- The pulse analysis discovered that it is possible to separate pulses caused by secondary muons from nucleons. Overall, the accurate pulse analysis could potentially be used for making a stand-alone neutron monitor as a particle spectrometer, which can be very valuable for analysis of solar energetic particle events.

\section{Acknowledgments}

We thank the personnel of Concordia station for hosting DOMC and DOMB neutron monitors and acknowledge hospitality of the Italian polar program PNRA (via the LTCPAA PNRA 2015/AC3 
and the BSRN PNRA OSS-06 projects) and the French Polar Institute IPEV. The work is supported by the Academy of Finland (projects CRIPA-X No. 304435, ESPERA No. 321882, QUASARE 330064, HEAIM-2 330427), and Finnish Antarctic Research Program (FINNARP).

\section{References}

[1] J.A. Simpson, The cosmic ray nucleonic component: The invention and scientific uses of the neutron monitor - (keynote lecture), Space Sci. Rev. 93 (2000) 11.

[2] H. Moraal, A. Belov and J. Clem, Design and co-ordination of multi-station international neutron monitor networks, Space Sci. Rev. 93 (2000) 285.

[3] H. Debrunner and U. Walther, Multiplicity measurements on the IGY neutron monitor at Jungfraujoch, Canadian Journal of Physics Supplement 46 (1968) 1140.

[4] Y.V. Balabin, B.B. Gvozdevsk, E.A. Maurchev, E.V. Vashenyuk and D.D. Dzhappuev, Fine structure of neutron multiplicity on neutron monitors, Astrophysics and Space Sciences Transactions 7 (2011) 283.

[5] S. Poluianov, I. Usoskin, A. Mishev, H. Moraal, H. Kruger, G. Casasanta et al., Mini Neutron Monitors at Concordia Research Station, Central Antarctica, J. Astron. Space Sci. 32 (2015) 281.

[6] D.T. Strauss, S. Poluianov, C. van der Merwe, H. Krüger, C. Diedericks et al., The mini-neutron monitor: a new approach in neutron monitor design, J. Space Weather Space Clim. 10 (2020) 39.

[7] J. Clem and L. Dorman, Neutron monitor response functions, Space Sci. Rev. 93 (2000) 335.

[8] Geant4 collaboration collaboration, Geant4 - a simulation toolkit, Nucl. Instr. Meth. Phys. A 506 (2003) 250.

[9] J.M. Picone, A.E. Hedin, D.P. Drob and A.C. Aikin, Nrlmsise-00 empirical model of the atmosphere: Statistical comparisons and scientific issues, J. Geophys. Res.: Space Phys. 107 (2002) SIA 15.

[10] E. Asvestari, A. Gil, G.A. Kovaltsov and I.G. Usoskin, Neutron Monitors and Cosmogenic Isotopes as Cosmic Ray Energy-Integration Detectors: Effective Yield Functions, Effective Energy, and Its Dependence on the Local Interstellar Spectrum, J. Geophys. Res. Space Phys. 122 (2017) 9790.

[11] S.V. Poluianov, I.G. Usoskin, A.L. Mishev, M.A. Shea and D.F. Smart, GLE and Sub-GLE Redefinition in the Light of High-Altitude Polar Neutron Monitors, Solar Phys. 292 (2017) 176 [1711.06161]. 